\title{
EXPERIMENTAL AND NUMERICAL STUDIES ON WAVE TRANSFORMATION OVER ARTIFICIAL REEFS
}

\author{
Shan-Hwei Ou ${ }^{1}$, Tai-Wen Hsu ${ }^{2 *}$, Jian-Feng Lin ${ }^{2}$, Jian-Wu Lai ${ }^{3}$, Shih-Hsiang Lin ${ }^{2}$, Chen- \\ Chen Chang ${ }^{2}$, Yuan-Jyh Lan ${ }^{2}$
}

\begin{abstract}
A laboratory measurement on the flow field, turbulence and wave energy of spilling breakers over artificial reefs is presented. Instantaneous velocity fields of propagating breaking waves on artificial reefs were measured using Particle Image Velocimeter (PIV) and Bubble Image Velocimeter (BIV). Variations of water surface elevation were observed by using Charge Coupled Device (CCD) cameras with horizontal posture. The experimental results showed that the initial bubble velocity in the aerated region is faster than phase speed with a factor of 1.26 . The velocity profiles are identical to the shallow water theory. It is found that a low flow velocity exists due to an opposite but equal onshore and offshore velocity. Significant turbulent kinetic energy and turbulent Reynolds stress are produced by breaking waves in the front of aerated region, then move offshore and decay. The calculated total energy dissipation rate was compared to that based on a bore approximation.
\end{abstract}

Keywords: DIP, PIV, BIV, Breaking wave

\section{INTRODUCTION}

Hard engineering coastal structures such as coastal dyke, offshore breakwaters and groin are built to protect the coastal line. However, these structures have disadvantages, such as coastal erosion by improper design that accelerate disappearance of sand, decrease of attraction and harmonious with environment. Along with the rising of environmental consciousness in recent years, the hard engineering methods are not only solutions to coastal defense. The flexible working methods are now becoming alternate solutions such as submerged breakwater, artificial nourishment and artificial reefs. Among them, artificial reefs have high potential in practical applications because they are acting like natural reefs. In the past, many researchers investigated the interaction between wave and structures through numerical or experimental test. In the last decade, non-intrusive measurement techniques were employed to observe the various complex flow phenomena under breaking waves over coastal structures. The laser Doppler velocimetry (LDV) and particle image velocimetry (PIV) technique were successfully used to measure breaking wave flow field in a surf zone. Ting and Kim (1994) applied the LDV to observe the vortex generation in water waves propagation over a submerged obstacle, and compared the scale of vortex by K-C number.They found that the scale of the vortex above the structure surface was affected by the K-C number. Petti et al. (1994) investigated the wave velocity field measurement over a submerged breakwater by PIV method, and discussed the variations of vortex under the wave breaking condition. Chang el al.(2001) used the experiment and numerical simulation to investigated vortex generation and evolution in water waves propagating over a submerged rectangular obstacle, and measured the flow field around the submerged obstacle by PIV method.

In wave breaking condition, Yasuda el at. (1997) investigated the flow field and their breaker types by simulating the solitary wave propagation over a submerged obstacle. Jansen (1986) used the fluorescent dye and ultraviolet light to measure displacement of the particles in aerated region. However, the results suffered from poor resolution for spatial variation. Chang and Liu (1998) used the PIV to measure the maximum velocity and associated acceleration and vorticity of the overturning jet of a breaking wave. Unfortunately,PIV is limited in the case where breaking wave produced the bubbles and thus scattered the laser light. PIV is only valid in the water region, therefore the instruments were limited by the non-aerated region. Greated and Emarat (2000), Ting and Kirby (1994, 1995), Perlin et al. (1996) used the LDV (Laser Doppler Velocimetry) to measure the aerated region, but it is only for single-point measurement. To overcome air bubble effect on the measurement Hassan et al. (1998), Nishino et al. (2002) and Lindken and Merzkirch (2002), applied the 'shadowgraphy' method to to measure bubble velocity by correlating bubbles or tracking each bubble in the recorded images. Govender et al., (2002) and Ryu et al. (2005) also have successfully measured the velocity field in the aerated region and in the overtopping region with air bubble entrainment. In this study, the mechanism of two-dimensional flow field of spilling breakers

\footnotetext{
${ }^{1}$ Department of Environmental Resources Management, Tajen University, 20, Weisrin Rd., Yanpu, Pingtung 907, Taiwan.

${ }^{2}$ Department of Hydraulic \& Ocean Engineering, National Cheng Kung University, 1, University Road, Tainan 701, Taiwan, corresponding author.

${ }^{3}$ Department of Civil Engineering, Nagoya University, Furo-cho, Chikusa-ku, Nagoya 464-8603, JAPAN.
} 
on an artificial reef was investigated experimentally using bubble image velocimetry.

\section{EXPERIMENTAL FACILITIES AND SETUP}

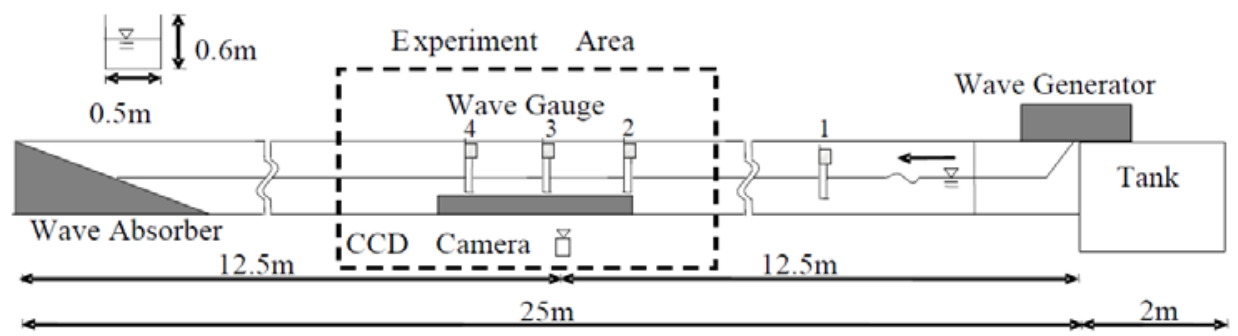

Figure 1. Experimental facilities and setup.

The experiments were conducted in a wave flume at Department of Hydraulic and Ocean Engineering of National Cheng Kung University. The wave tank is $25 \mathrm{~m}$ long, $0.5 \mathrm{~m}$ wide and $0.6 \mathrm{~m}$ high. The wave maker is of piston type installed at one end of the wave tank and controlled by a computer. The wave absorber is at the other end of the tank to absorb the wave energy and reduce reflection. The water depth was kept constant at $h=0.165 \mathrm{~m}$ high. A rectangular model structure is 2.4 $\mathrm{m}$ long and $0.115 \mathrm{~m}$ high. The wave data were recorded at a sampling rate of $100 \mathrm{~Hz}$ by capacitancetype wave gauges distributed at 4 fixed locations. Fig. 1 is a schematic diagram of the facilities and apparatus layouts.

The PIV system used in the present study includes a dual-head pulsed laser, laser light sheet optics, a CCD camera, and a synchronizer. The dual-head pulsed laser is Nd: YAG laser that has a 20 $\mathrm{Hz}$ repetition rate and $120 \mathrm{~mJ} /$ pulse maximum energy output. It was used as the PIV illumination source. Images were recorded using a 12-bit CCD camera that has a $1600 \times 1200$ pixel resolution and 30 frames per second (fps) maximum framing rate. The BIV system used in the present study includes a high speed camera, and two 600W light bulbs. The images were captured by IDT MotionProX3TM PLUS high speed camera. The camera has a resolution of $1280 \times 1024$ pixels and a maximum framing rate of $2000 \mathrm{fps}$.

The water in the wave flume was seeded with nearly neutrally buoyant hollow glass spheres particles (TSI, normal mean diameter: 8-12 $\mu \mathrm{m}$; density: $1.05-1.15 \mathrm{~g} / \mathrm{cm}^{3}$ ) to enhance illumination efficiency during the PIV measurements. Because the wave-breaking region in the surf zone is too large to be captured in one single frame, the complete spatial distribution of velocities was integrated by the mosaic of frame from 5 fields of view (FOV), as shown in Fig2. Note that the origin $x=0$ is at the structure front wall, and the size of each FOV is shown in Table 1 and Table 2.

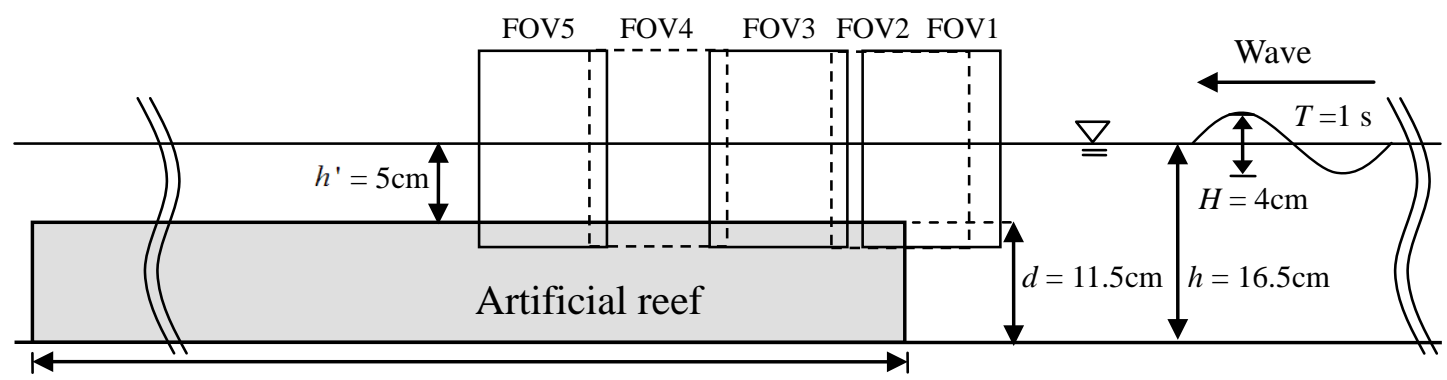

Figure 2. Five fields of view (FOV). 


\begin{tabular}{|l|l|l|l|}
\hline \multicolumn{4}{|c|}{ Table 1. The boundaries of FOV of the PIV } \\
\hline & Border-left (cm) & Border-right (cm) & \\
\hline FOV1 & -12.5 & 13.5 & PIV \\
FOV2 & -21 & 5 & PIV \\
FOV3 & -38 & -12 & PIV \\
FOV4 & -55 & -29 & PIV \\
FOV5 & -71.5 & -46.5 & PIV \\
\hline
\end{tabular}

\begin{tabular}{|l|l|l|l|}
\hline \multicolumn{4}{|c|}{ Table 2. The boundaries of FOV of the BIV } \\
\hline & Border-left (cm) & Border-right (cm) & \\
\hline FOV1 & -12.5 & 8.5 & \\
FOV2 & -21 & 0 & BIV \\
FOV3 & -38 & -17 & BIV \\
FOV4 & -55 & -34 & BIV \\
FOV5 & -71.5 & -50.5 & BIV \\
\hline
\end{tabular}

\section{VALIDATION OF THE BIV METHOD}

Because there was no particular BIV measurement system software package, the validation used the open source software created by Nobuhito Mori. Originally MPIV program was designed to analyze PIV. With modification of Mori's MPIV program,the software can be applied to analyze BIV as well. In the present study, the validation of the accuracy of this software is performed by the captured bubble image as shown in Fig. 3. After sampling 10 sets of images, the velocity calculating by modified MPIV is and from the images directly. The velocity was calculated using modified MPIV referred as $U$, and from the images directly is referred as $U_{R}$. Comparison between each velocity measurement was made to validate the BIV techniques. The result of the validation of the BIV method was shown in Fig. 4. The horizontal axis represents the time interval images; the vertical axis indicates the actual value of the error percentage. The mean error is about $2.10 \%$ and the maximum error is about $3.89 \%$. It proved the accuracy of the bubble velocity measurement.

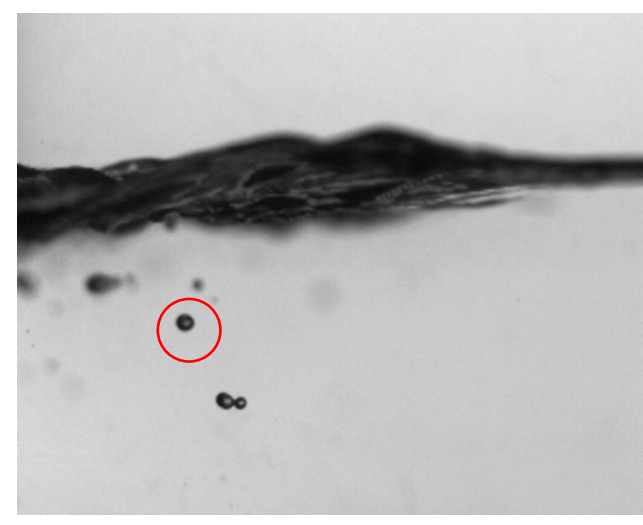

(a)

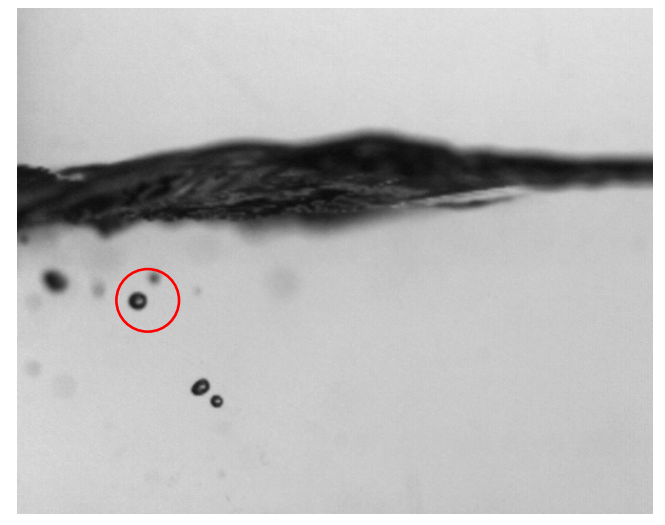

(b)

Figure 3. One set of captured bubble images. The time interval between two images is $0.001 \mathrm{~s}$. 


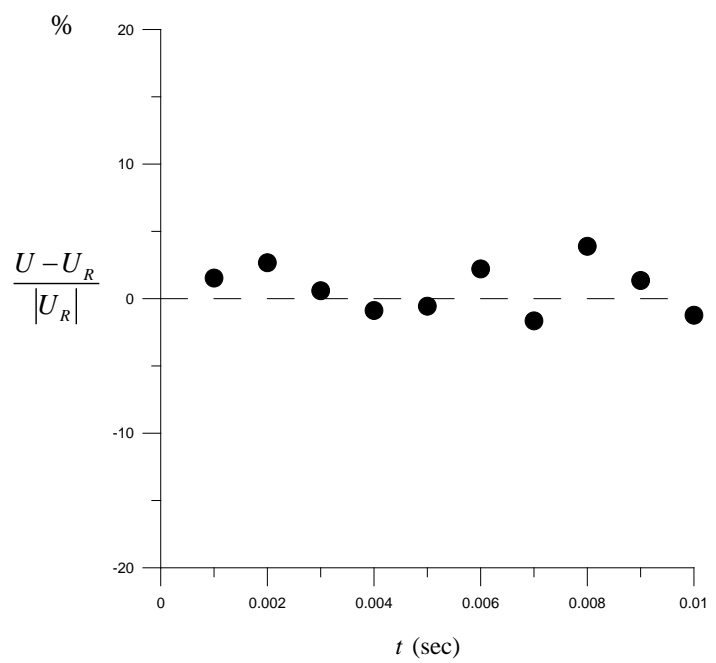

Figure 4. The error distribution of the BIV method.

\section{RESULTS AND DISCUSSION}

In the present study, the incident wave period is $1 \mathrm{~s}$ and wave height is $4 \mathrm{~cm}$. There were four wave gauges located at $8 \mathrm{~m}, 0 \mathrm{~m},-0.75 \mathrm{~m}$ and $-1.75 \mathrm{~m}$ from the front wall of the test model. Considering the test region and the resolution of the CCD camera, the test region was divided into five FOVs. Fig.5 shows that the surface elevation measured at $x=8 \mathrm{~m}$ from the front wall of the test model. The figure shows the wave become steady at the eighth wave, so the images are captured from the eighth to eleventh waves. The waves are not affected by the reflection of the slope at the end of the water tank and the secondary reflection from the panel of the wavemaker.

Fig. 6 shows the result of combined flow field of five regions as mentioned above. At $t=1 / 10 \mathrm{~T}$ the breaking waves pass the tip of the artificial reef, the velocity near the free surface is larger than the velocity near the structure. It is shown that the flow field in the upper layer is slightly affected by the velocity of wave trough in offshore direction. Because of the offshore velocity, the flow field in the lower layer forms a convergent stagnation point, and the velocity approaching to zero. At $t=4 / 10 \mathrm{~T}$, the wave form reached its stability value and started to break. From $t=5 / 10 \mathrm{~T}$ to $t=10 / 10 \mathrm{~T}$, the waves are broken and the water body affected by the gravity trapping air inside it which also causes resistance to the flow. In the aerated region near the structure of the lower layer, stagnation area occurs which obviously different from the upper layer near the free surface. As time increased, the stratification phenomena area become more obvious and the stagnation area becomes larger as losing its momentum caused by bubble collision during the wave breaking.

In Fig. 7 the color map stands for the $\mathrm{u} / \mathrm{C}$ value, $\mathrm{u}$ means horizontal flow velocity and $\mathrm{C}$ means theoretical wave velocity. When $\mathrm{u} / \mathrm{C}$ value is larger than 1 , the wave starts to break. At $\mathrm{t}=4 / 10 \mathrm{~T}$, the $\mathrm{u} / \mathrm{C}$ value is larger than 1 , so the wave starts to break. The maximum value of $\mathrm{u} / \mathrm{C}$ can reach about 2 at $\mathrm{t}=5 / 10 \mathrm{~T}$, and most of the values can reach 1.4 1.8. At $\mathrm{t}=6 / 10 \mathrm{~T}$ to $\mathrm{t}=10 / 10 \mathrm{~T}$, the $\mathrm{u} / \mathrm{C}$ value decrease rapidly in the aerated area. After wave breaks, the wave trap air into the water and affected by the front and bottom water body. The velocity starts to decrease, and the $\mathrm{u} / \mathrm{C}$ value closes to 1 . 


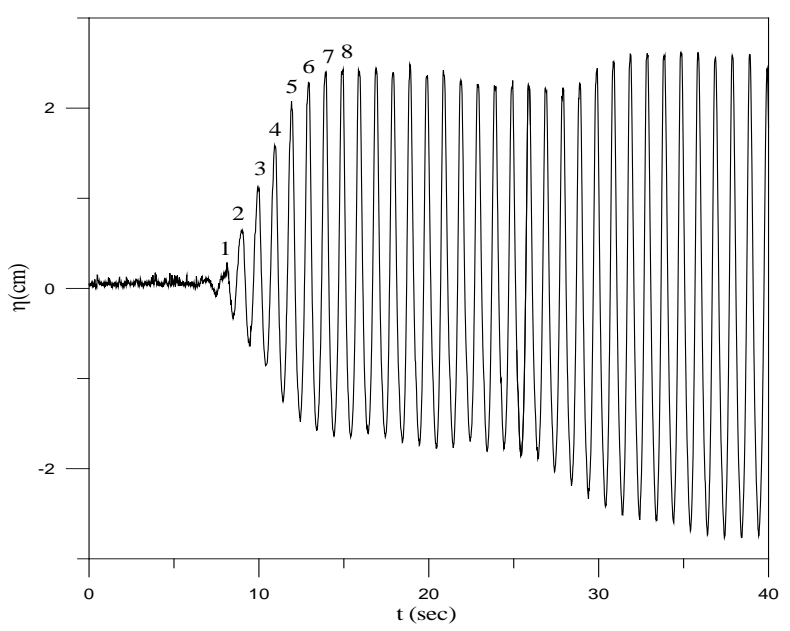

Figure 5 .The surface elevation measured at $x=8 \mathrm{~m}$ from the front wall of the test model.
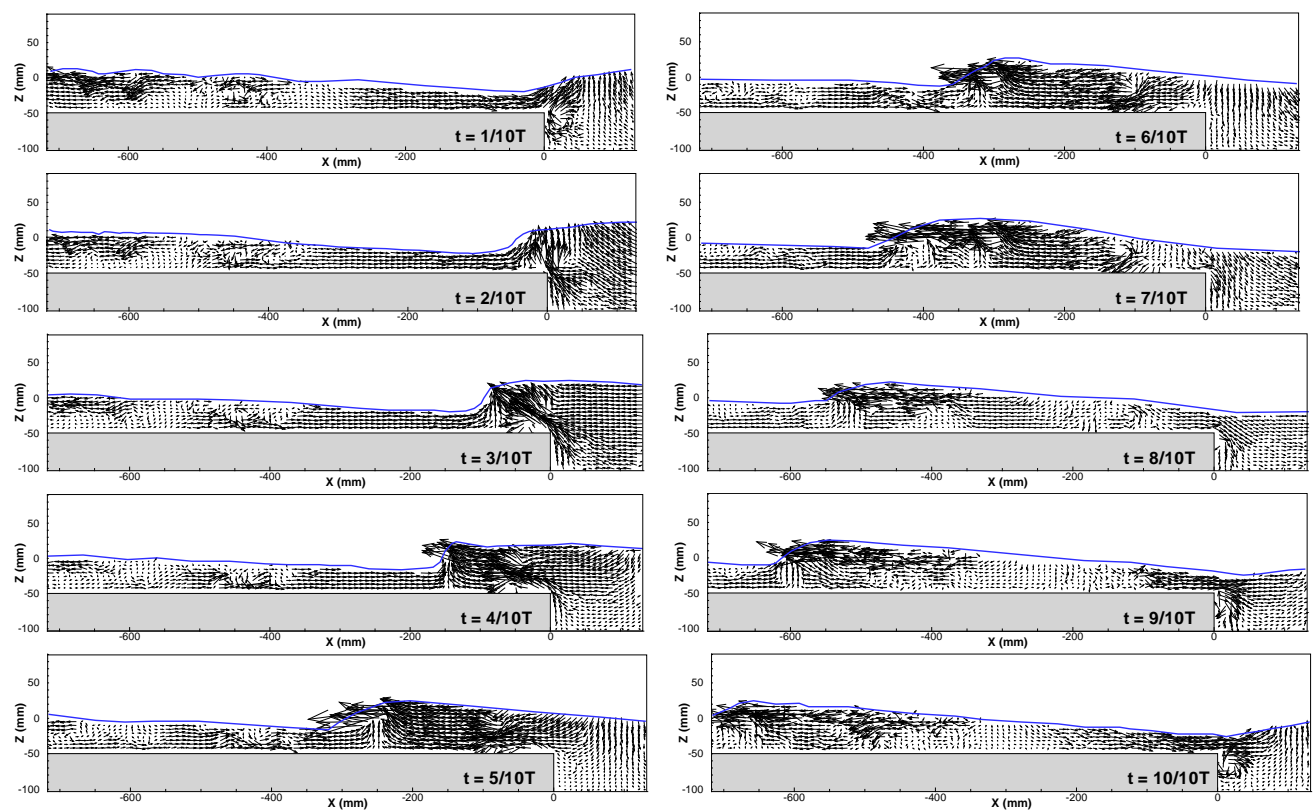

Figure 6. The combined flow field measured by PIV and BIV. 


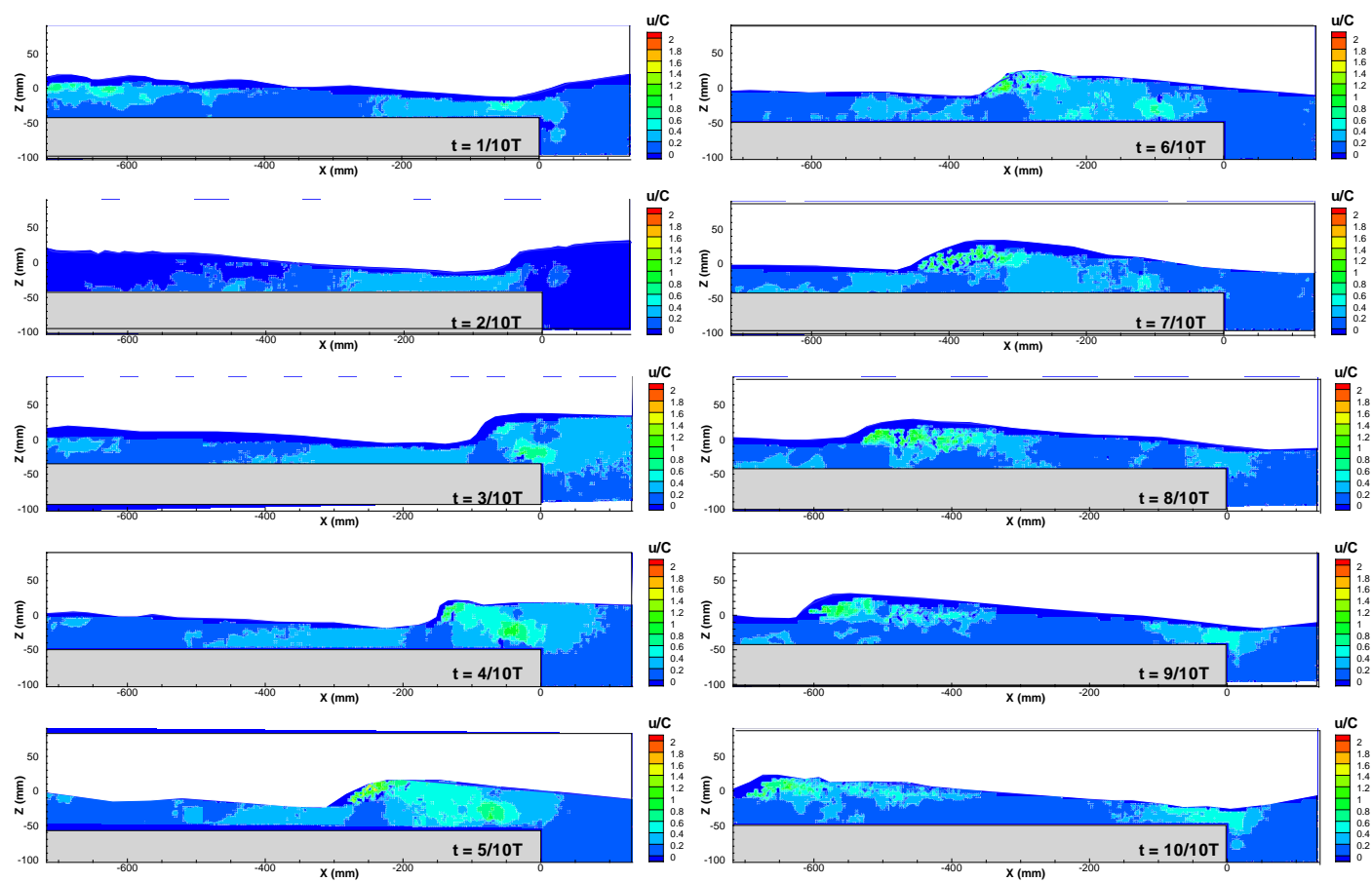

Figure 7. The combined flow field of $u / C$.

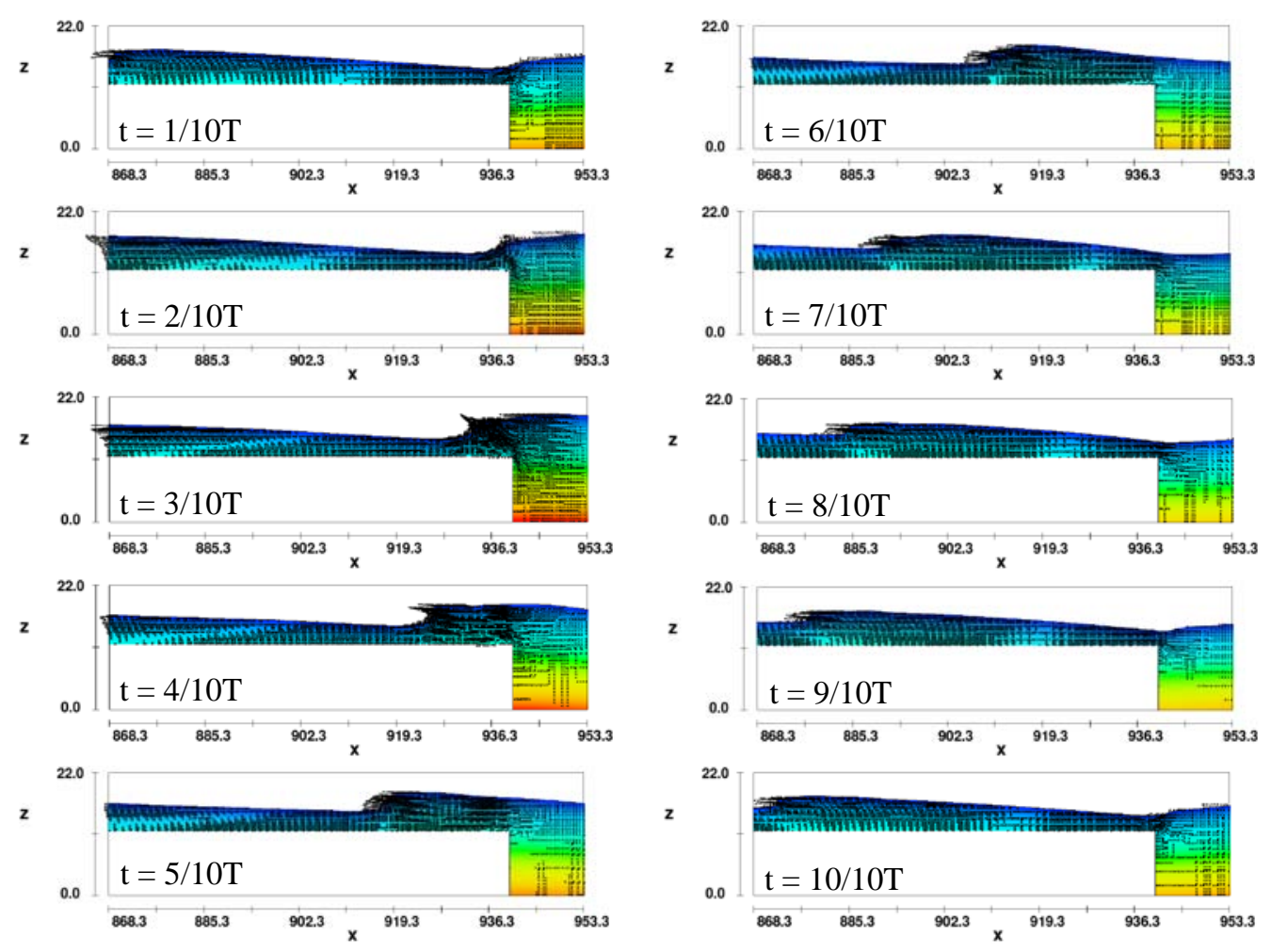

Figure. 8 The numerical results calculated by Flow 3D.

The numerical result calculated by Flow 3D was shown in Fig. 8. At $t=7 / 10 \mathrm{~T}$ the stagnation area was occurred and move with wave flow. At $t=2 / 10 \mathrm{~T}$ to $\mathrm{t}=6 / 10 \mathrm{~T}$ the stratification phenomena become more obvious, the numerical results show good agreement with experimental results. 


\section{CONCLUSION}

In this study, bubble image velocimetry and particle image velocimetry combining with highspeed CCD cameras and other devices are usedto record the interaction between wave breaking and artificial submerged reef model. The wave transformations over artificial reef are investigated. The wave elevation measured by the wave gauge above the artificial reef shows the wave height decay obviously when wave breaks above the artificial reef. After the wave breaking, there was a stratification between the water body near the surface and the structure, and the latter has a smaller velocity. As time increased, the bubble range became narrow and the stratification grew into more obvious. When the wave is breaking, the bubble has the maximum velocity. The velocity within the aerated area is affected by the front and bottom water body, the $\mathrm{u} / \mathrm{C}$ value form 2 decrease to 1.2 1.4, then declines to about 1 . Both experimental and numerical results showed the wave height decayed after the wave breaking and it became more obvious as the distance further from the wave breaking.

\section{REFERNCES}

Chang, K. A. and Liu, P. F., 1998, Velocity, Acceleration and Vorticity under a Breaking Wave, Physical of Fluids, Vol. 10, pp. 327-329.

Chang, K. A., Hsu, T. J. and Liu, P. F., 2001, Vortex Generation and Evolution in Water Waves Propagating over a Submerged Rectangular Obstacle, Part I. Solitary Waves, Coastal Engineering, Vol. 44, pp. 13-36.

Greated, C. A. and Emarat, N., 2000, Optical Studies of Wave Kinematics, Advances in Coastal and Ocean Engineering, Vol. 6, ed P L-F Liu (Singapore: World Scientific) pp 185-223.

Govender, K., Mocke, G.P. and Alport, M.J., 2002, Videoimaged surf zone wave and roller structures and flow fields, Journal of Geophysical Research, 107(C10), 3177.

Hassan, Y. A., Schmidl, W. D. and Ortiz-Villafuerte, J., 1998, Investigation of Three-dimensional Two-phase Flow Structure in a Bubbly Pipe, Measurement Science Technology, Vol. 9, pp.309326.

Jansen, P. C. M., 1986, Laboratory Observations of the Kinematics in the Aerated Region of Breaking Waves, Coastal Engineering, Vol.9, pp. 453-477.

Lindken, R. and Merzkirch, W., 2002, A Novel PIV Technique for Measurements in Multi-Phase Flows and Its Application to Two-Phase Bubbly Flows, Experiments in Fluids, Vol. 33, pp.814825.

Nishino, K., Kato, H. and Torii, K., 2000, Stereo Imaging for Simultaneous Measurement of Size and Velocity of Particles in Dispersed Two-Phase Flow, Measurement Science Technology, Vol. 11, pp.633-645.

Perlin, M., He, J. and Bernal, L. P., 1996, An Experimental Study of Deep Water Plunging Breakers, Physics of Fluids, Vol. 8, pp.2365-2274.

Petti, M., Quinn, P. A., Liberatore, G.. and Easson, W. J., 1994, Wave Velocity Field Measurement over a Submerged Breakwater, Proceeding 24th International Conference Coastal Engineering, Japan, ASCE, pp. 525-539.

Ryu, Y., Chang, K. A. and Lim, H. J., 2005, Use of Bubble Image Velocimetry for Measurement of Plunging Wave Impinging on Structure and Associated Greenwater, Measurement Science and Technology, Vol. 16, pp.1945-1953.

Takashi, Y., Hidenmi, M. and Natsuki, M., 1997, Kinematics of Overturning Solitary Waves and Their Relations to Breaker Types, Coastal Engineering, Vol. 29, pp. 317-346.

Ting, F. C. K. and Kim, Y. K., 1994, Vortex Generation in Water Waves Propagation over a Submerged Obstacle, Coastal Engineering, Vol. 24, pp.23-49.

Ting, F. C. K. and Kirby, J. T., 1994 Observation of Undertow and Turbulence in a Laboratory Surf Zone, Coastal Engineering, Vol. 24, pp.51-80.

Ting, F. C. K. and Kirby, J. T., 1995, Dynamics of Surf-Zone Turbulence in a Strong Plunging Breaker, Coastal Engineering, Vol. 24, pp.177-204.

Yasuda, T., Mutsuda, H. and Mizutani, 1997, N., Kinematics of overturning solitary waves and their relations to breaker types, Coastal Engineering, pp. 317-346. 